\title{
Nile Red and 2-NBDG Are Incompatible for the Simultaneous Detection of Lipid and Glucose Accumulation
}

\author{
Andrew M. Hogan, Viswanathan Swaminathan, Nikitha K. Pallegar, and Sherri L. Christian \\ Department of Biochemistry, Faculty of Science, Memorial University of Newfoundland, 232 Elizabeth Ave, \\ St. John's, NL, Canada A1B $3 X 9$ \\ Correspondence should be addressed to Sherri L. Christian; sherri.christian@mun.ca
}

Received 14 October 2016; Revised 22 November 2016; Accepted 27 November 2016

Academic Editor: Jaan Laane

Copyright (C) 2016 Andrew M. Hogan et al. This is an open access article distributed under the Creative Commons Attribution License, which permits unrestricted use, distribution, and reproduction in any medium, provided the original work is properly cited.

\begin{abstract}
Glucose is the universal energy source and a critical substrate for lipid synthesis in mammalian cells. Analysis of both glucose and lipid in cells is important for the understanding of the regulation of lipid synthesis in many cell types, but especially adipocytes, the major storage cell for fat in mammals. The fluorescent 7-nitrobenz-2-oxa-1,3-diazole (NBD) derivative of glucose, 2-NBDG, is used to monitor glucose uptake and the lipid-selective fluorophore Nile red is used to monitor lipid accumulation. Previous reports have used NBD-based fluorophores and Nile red simultaneously despite the possibility of spectral overlap. In this study, we determined if these fluorophores were experimentally compatible in preadipocytes and adipocytes stained with 2-NBDG and Nile red separately or costained. We found that Nile red is detectable in the wavelengths necessary to excite and detect 2-NBDG. This interference was further increased by the solvatochromic effect of lipid-localized Nile red. In addition, we found a synergistic increase in fluorescent intensity when both fluorophores were present. Unfortunately, even fine control of the excitation or emission wavelengths did not identify wavelengths suitable for selective detection when cells were costained. Therefore, 2-NBDG and Nile red cannot be used simultaneously_but can likely be used sequentially_to assess glucose uptake and lipid accumulation in lipid-laden cells.
\end{abstract}

\section{Introduction}

Lipids accumulate in mammalian cells either through extraction of dietary lipids from the circulation or by de novo lipogenesis using precursor molecules, of which glucose is a primary and essential substrate $[1,2]$. While this process occurs in all mammalian cells, adipocytes are the predominant cellular constituent of adipose tissue where excess energy is stored as neutral lipids in the form of triacylglycerols and cholesteryl esters. The study of lipogenesis as well as adipogenesis, which is the development of mature adipocytes from preadipocytes, has had a renewal in interest due to the rapid rise in obesity globally over the past 2 decades $[3,4]$.

Differentiation of preadipocytes to mature adipocytes is characterized by the accumulation of lipid over the course of 1-2 weeks in vitro where the cell progresses through multiple stages characterized by key changes to gene expression [5]. Understanding the regulation of these processes is often dependent on measuring both lipid accumulation and cellular glucose uptake. A common cellular model used to study adipocyte biology is the 3T3-L1 cell line, which is murine embryonic preadipocytes capable of differentiating in vitro into lipid-laden mature adipocytes [6,7].

The use of fluorescent glucose analogues has recently come into favour as a replacement for radiolabeled glucose for monitoring glucose uptake, following the general trend away from radioisotopes [8-10]. D-glucose is modified by the covalent attachment of the 7-nitrobenz-2-oxa-1,3-diazolyl fluorophore to create 2-NBDG, a modification which has only minor effects on its uptake properties as, similar to D-glucose, uptake of 2-NBDG occurs through glucose transporters (GLUTs) and with comparable $K_{M}$ values to D-glucose [11]. Typical excitation and emission wavelengths for detecting 2NBDG (and other NBD-based fluorophores) are approximately $480 \mathrm{~nm}$ and $550 \mathrm{~nm}$, respectively [11,12].

First applied over 30 years ago as a hydrophobic stain to visualize lipid droplets, Nile red is now recognized to stain neutral, polar, and charged lipids, and hydrophobic protein 
surfaces [13-15]. Nile red is an affordable lipid stain that is both widely available and extensively characterized. The basis of Nile red detecting lipid lies in solvatochromism, as the fluorescence emissions shift from red $(630 \mathrm{~nm})$ to green-yellow $(580 \mathrm{~nm})$ as the environment becomes more hydrophobic $[13,16]$. Taking this into account, Nile red is typically excited and detected in the broad ranges of $460-$ $560 \mathrm{~nm}$ and $530-630 \mathrm{~nm}[13,15,17]$.

Previously, NBD-cholesterol and NBD-sphingomyelin have been used simultaneously with Nile red to identify intracellular lipid droplets, suggesting that 2-NBDG and Nile red could also be used simultaneously to monitor glucose intake and lipid abundance $[12,17,18]$. However, an experimental validation of the suitability of these fluorophores to retain their selectivity when used simultaneously has not been reported. As there is substantial, but not complete, overlap in the individual fluorescence spectra of NBD and Nile red, the potential for nonselective detection of these fluorophores has been noted previously [19]. As of yet, there are no reports on the simultaneous use of the NBD glucose analogue, 2-NBDG, with Nile red. Here, we sought to determine if 2-NBDG and Nile red could be used simultaneously to selectively detect glucose uptake and lipid accumulation, respectively. Using lipid-laden 3T3-L1 adipocytes as a model, we found that, in the presence of intracellular lipid, Nile red was detected with the same excitation and emission wavelengths ideal for detecting 2-NBDG. In fact, we were unable to identify any excitation/emission pair that was selective for 2-NBDG in the presence of Nile red-stained lipid. Furthermore, we observed a significant increase in fluorescence emission intensity when both 2-NBDG and Nile red were used to costain lipid-laden mature adipocytes. Thus, we conclusively demonstrate that 2-NBDG and Nile red cannot be used simultaneously to specifically measure glucose uptake and lipid accumulation.

\section{Materials and Methods}

2.1. Cell Culture. The murine embryonic preadipocyte cell line 3T3-L1 (ATCC CL-173) was cultured and differentiated as reported previously [20]. All media and supplements were purchased from Life Technologies unless otherwise indicated. In brief, the cells were grown at $37^{\circ} \mathrm{C}$ and $5 \%$ $\mathrm{CO}_{2}$ in high-glucose DMEM supplemented to $10 \%$ newborn calf serum, $1 \mathrm{mM}$ sodium pyruvate, $100 \mathrm{U} / \mathrm{mL}$ penicillin, and $100 \mu \mathrm{g} / \mathrm{mL}$ streptomycin. To differentiate preadipocytes into lipid-laden adipocytes, cells (at passage 15 or earlier) were plated in wells of a 96-well plate at a density of 2.2 $\times 10^{4} \mathrm{cells} / \mathrm{cm}^{2}$. Two days after achieving confluence, the cells were initiated to differentiate in high-glucose DMEM supplemented to $10 \%$ fetal calf serum, $1 \mathrm{mM}$ sodium pyruvate, $100 \mathrm{U} / \mathrm{mL}$ penicillin, and $100 \mu \mathrm{g} / \mathrm{mL}$ streptomycin (complete medium) with $0.5 \mathrm{mM}$ isobutylmethylxanthine and $0.1 \mu \mathrm{M}$ dexamethasone (EMD Millipore). Two days later, the cells were incubated in complete medium containing $10 \mu \mathrm{g} / \mathrm{mL}$ recombinant human insulin (Sigma-Aldrich). The medium was replaced every 2 days with complete media until 5 days after insulin treatment when the cells had visually accumulated lipid.
2.2. 2-NBDG and Nile Red Staining. Cells were differentiated as above in a black $\mu$ Clear $^{\circledR}$ 96-well plate (Greiner BioOne). Differentiated lipid-laden adipocytes or undifferentiated preadipocytes were incubated in the dark with $10 \mu \mathrm{g} / \mathrm{mL}$ Nile red (Santa Cruz Biotechnology) and/or $100 \mu \mathrm{g} / \mathrm{mL} 2$ NBDG (Cayman Chemicals) in glucose-free, serum-free DMEM for 45 minutes at $37^{\circ} \mathrm{C}$ and $5 \% \mathrm{CO}_{2}$. Excess stain was removed with 5 washes of cold PBS $(137 \mathrm{mM} \mathrm{NaCl}$, $2.7 \mathrm{mM} \mathrm{KCl}, 10 \mathrm{mM} \mathrm{Na}_{2} \mathrm{HPO}_{4}, 1.8 \mathrm{mM} \mathrm{KH}_{2} \mathrm{PO}_{4}$, and $\mathrm{pH}$ 7.4). As a vehicle control, cells were also treated with the same volume of ethanol (vehicle for 2-NBDG) or dimethyl sulfoxide (vehicle for Nile red).

2.3. Fluorescence Microscopy. Immediately after staining, cells were visualized under a Leica DM IRE2 inverted fluorescence microscope equipped with a mercury arc lamp. A Retiga Exi camera (QImaging) was used to capture images exposed for $300 \mathrm{~ms}$ under bright field, the L5 filter cube (480/40 $\mathrm{nm}$ excitation, $527 / 30 \mathrm{~nm}$ emission), and the TX2 filter cube (560/40 nm excitation, $645 / 75 \mathrm{~nm}$ emission) using the 20x objective (N.A. 0.4). OpenLab v5.5.0 software package (Perkin Elmer) was used to operate the microscope and digitally capture the images. ImageJ v1.5 (National Institutes of Health) [21] was used to process images and obtain multicolour overlays.

2.4. Spectral Analysis. Immediately after staining, the cover of the plate was removed and the fluorescence was measured with a Synergy Mx fluorescent plate reader equipped with a xenon flash lamp (BioTek Instruments). Using excitation wavelengths fixed at $450 \mathrm{~nm}, 520 \mathrm{~nm}$, and $570 \mathrm{~nm}$, the emissions were collected in $3 \mathrm{~nm}$ intervals up to $700 \mathrm{~nm}$. Similarly, to generate excitation spectra the emissions were collected at the fixed wavelengths $520 \mathrm{~nm}, 580 \mathrm{~nm}$, and $650 \mathrm{~nm}$ in $3 \mathrm{~nm}$ intervals with excitation starting at $300 \mathrm{~nm}$. The slit width was set to $9 \mathrm{~nm}$ for the excitation and emission wavelengths. Data was compiled using the Gen5 v2.05 software package (BioTek Instruments).

2.5. Statistical Analysis. Statistical analysis was performed in R v3.3.0 [22]. To compare fluorescence data of singly and costained samples, a one-way ANOVA followed by a Tukey's post hoc test was performed. The data passed the ANOVA assumptions (normally distributed, homoscedastic, and equal sample variances) as per the Shapiro-Wilks test, the Breusch-Pagan test, and Levene's test, respectively. Expected costain fluorescence was calculated by summing the values from the singly stained samples of 2-NBDG and Nile red. This was compared to the experimental costain samples using an independent Student's $t$-test. Differences were considered statistically significant at $P<0.05$.

\section{Results}

Previous reports indicate that $2-\mathrm{NBDG}$ can be excited at approximately $480 \mathrm{~nm}$ and detected near $550 \mathrm{~nm}$ [11, 12]. Nile red can be excited and detected by much broader wavelengths, apparently between $460 \mathrm{~nm}$ and $560 \mathrm{~nm}$ and between $530 \mathrm{~nm}$ and $630 \mathrm{~nm}$, respectively [13, 15, 17]. We 

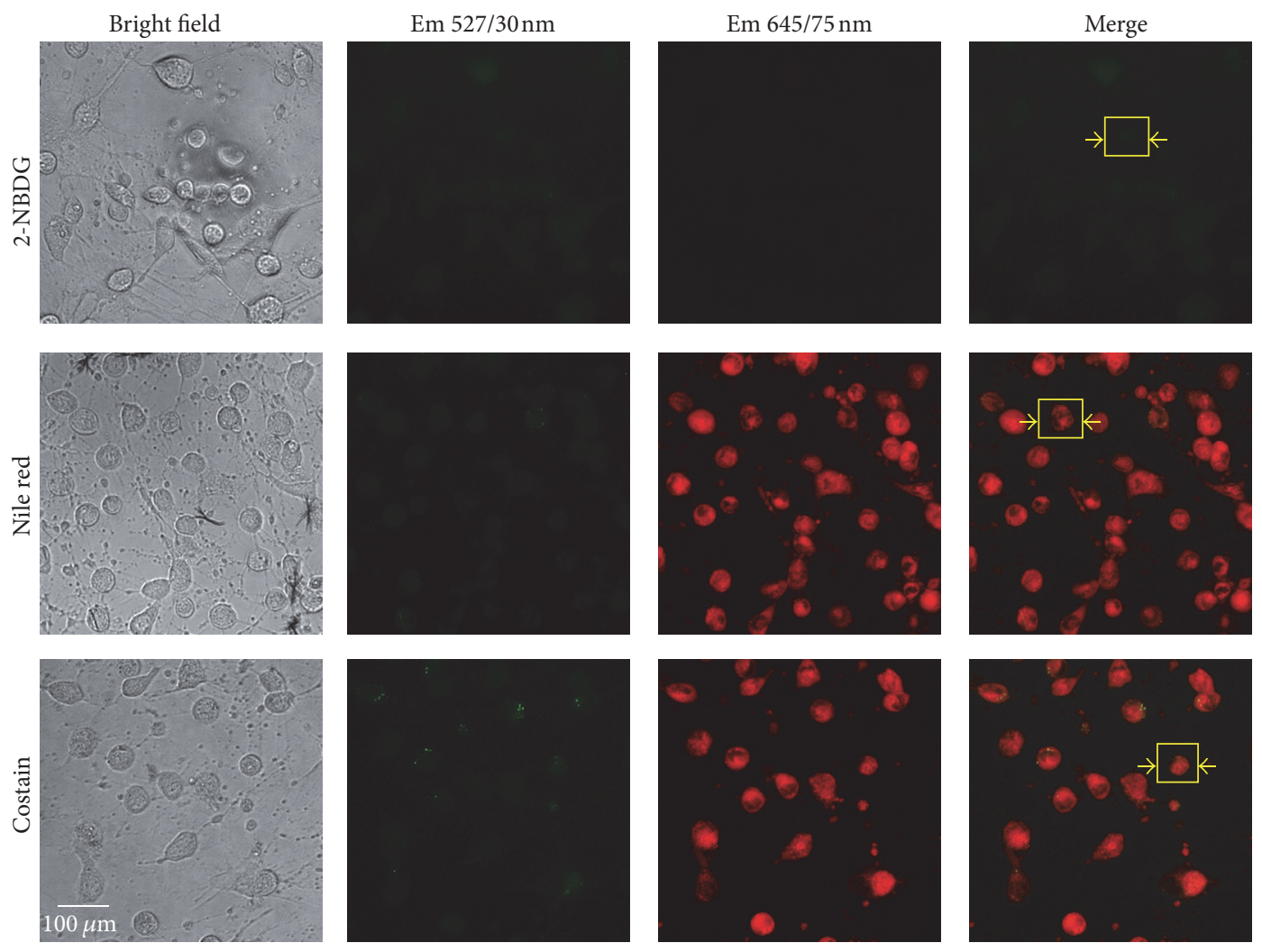

(a)
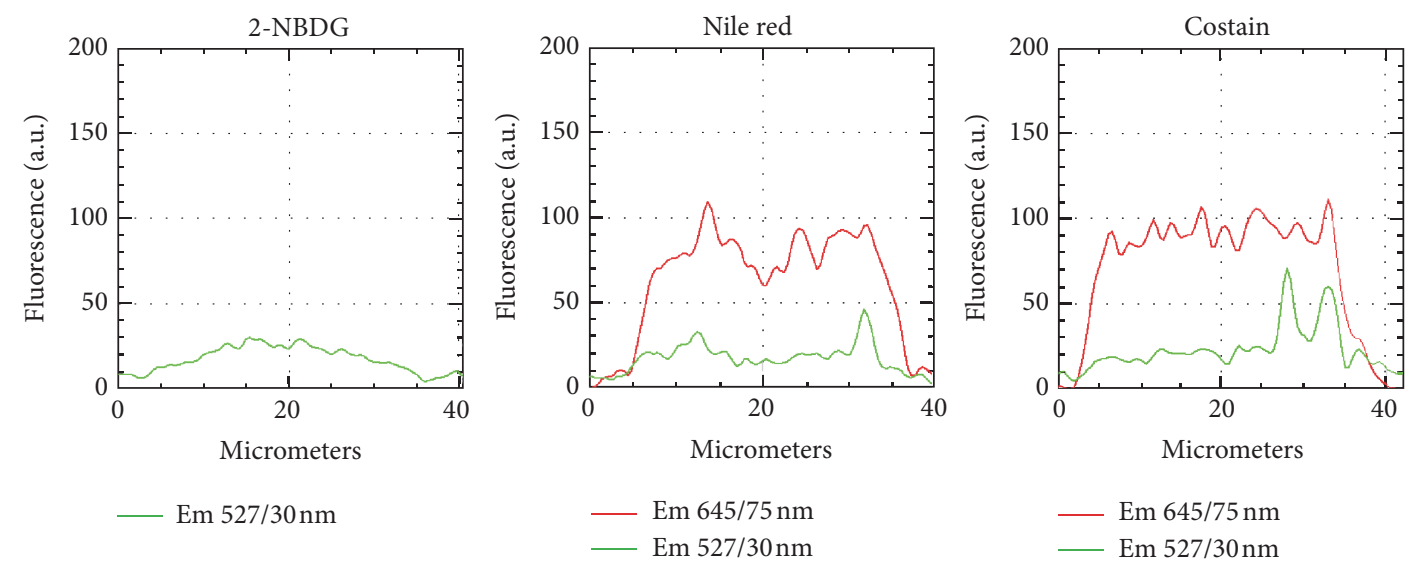

(b)

FIGURE 1: Fluorescence of 2-NBDG and Nile red in 3T3-L1 preadipocytes. (a) Fluorescence micrographs of unstained cells and cells stained with only 2-NBDG, only Nile red, or both simultaneously (costain) excited at $480 / 40 \mathrm{~nm}$ or $560 / 40 \mathrm{~nm}$ and detected with emissions at $527 / 30 \mathrm{~nm}$ or $645 / 75 \mathrm{~nm}$, respectively. (b) Representative plot profiles of the cells indicated with yellow arrowheads in (a) in the first panel showing the intensities across a single cell when emissions were collected at 527/30 nm and 645/75 nm. Representative images from $n=3$ experiments.

used fluorescence microscopy to assess the individual fluorescence of 2-NBDG and Nile red in cells stained with each fluorophore alone and in combination (Figure 1). In the preadipocytes, we found that 2-NBDG was detected weakly and diffusely in only the green channel (Ex $480 / 40 \mathrm{~nm}$, Em $527 / 30 \mathrm{~nm}$ ) (Figure 1(a)). We observed that Nile red was detected using both filter sets and at higher intensity in the red channel compared to the green channel, which is clearly shown by the plot profile (Figure 1(b)). However, in the presence of both fluorophores, the fluorescence microscopy images and the plot profiles appeared to be more similar to the cells stained with only Nile red (Figure 1).

Nile red is reported to be a lipid-selective stain and that its fluorescence shifts to shorter wavelengths when staining neutral lipid [15]. To determine if lipid stained by Nile red interferes with 2-NBDG fluorescence detection, we 

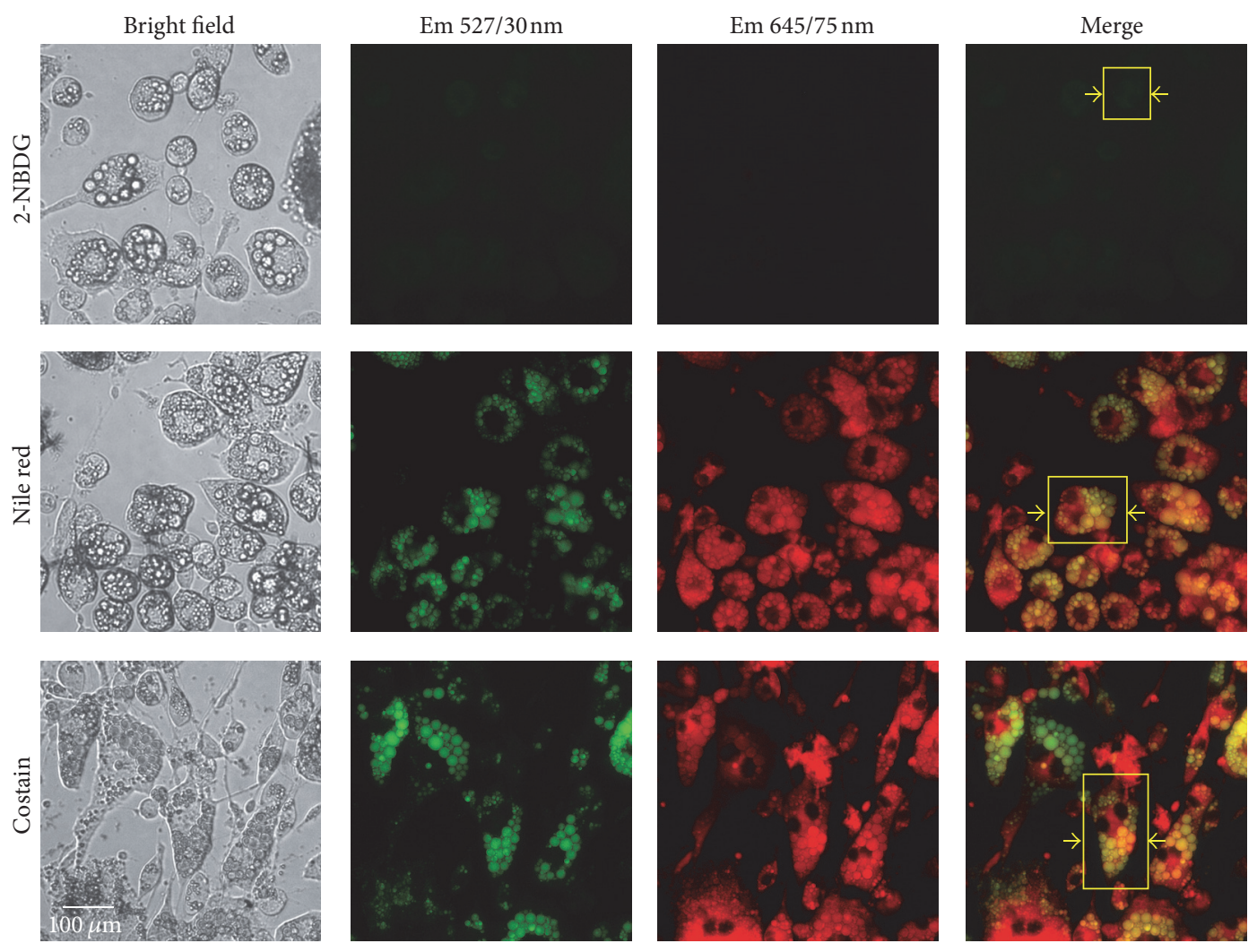

(a)
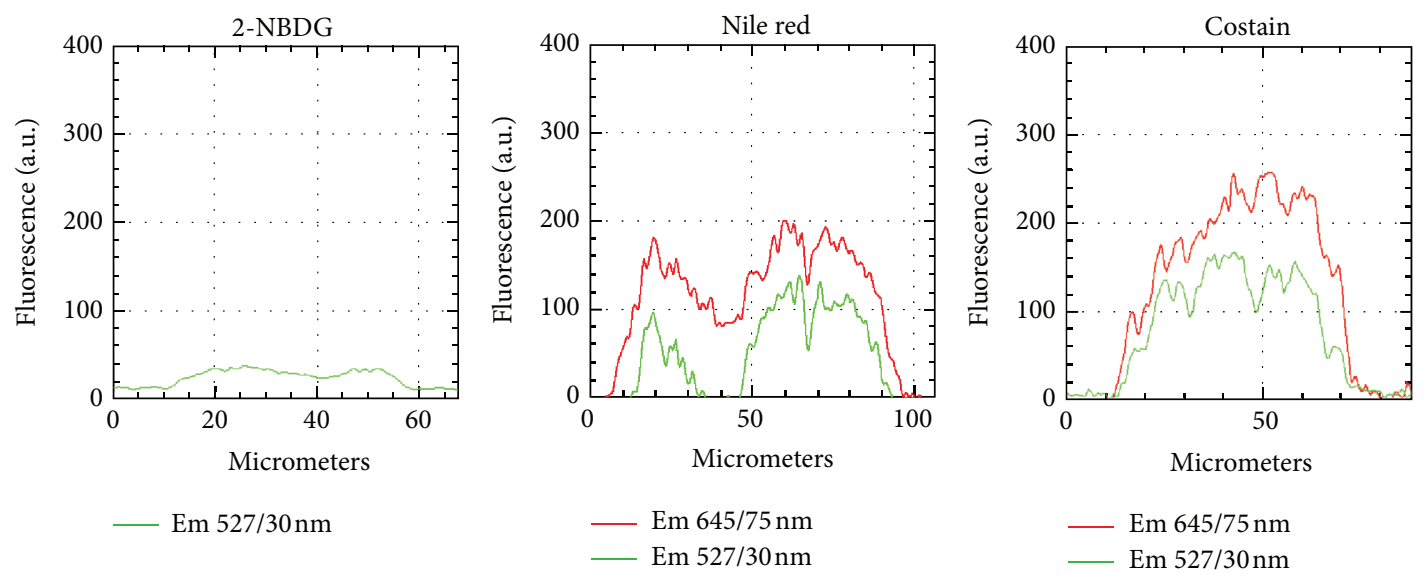

(b)

FIGURE 2: Fluorescence of 2-NBDG and Nile red in 3T3-L1 adipocytes. (a) Fluorescence micrographs of unstained cells and cells stained with only 2-NBDG, only Nile red, or both simultaneously (costain) excited at 480/40 nm or 560/40 nm and detected with emissions at $527 / 30 \mathrm{~nm}$ or $645 / 75 \mathrm{~nm}$, respectively. (b) Representative plot profiles of the cells indicated with yellow arrowheads in (a) in the first panel showing the intensities across a single cell when emissions were collected at 527/30 nm and 645/75 nm. Representative images from $n=3$ experiments.

next stained and visualized differentiated lipid-laden 3T3L1 adipocytes (Figure 2). We found that the lipid droplets in the adipocytes were selectively and brightly visualized in the green channel with Nile red staining (Figure 2(a)) and are apparent in the plot profiles as peaks (Figure 2(b)). In the red channel, we found that Nile red fluorescence was more diffuse but was still concentrated at the lipid droplets (Figure 2(a)). Moreover, the signals from the green and red channels have similar profiles, suggesting that Nile red detected many of the same structures and organelles in both channels (Figure 2(b)).

Given the observed spectral overlap of 2-NBDG and Nile red, we next analyzed cells stained with 2-NBDG and Nile red alone via spectrofluorometry to determine if we could refine the wavelength selection to selectively detect 2-NBDG and Nile red (Supplementary Fig. 1 
and Fig. 2 in Supplementary Material available online at http://dx.doi.org/10.1155/2016/5215086) [11, 13, 15]. We found that in all cells the excitation and emission maxima for 2NBDG were $480 \mathrm{~nm}$ and $550 \mathrm{~nm}$, respectively (Supplementary Fig. 1). Unlike 2-NBDG, Nile red fluorescence depended on the cell type as the excitation maximum in adipocytes was $520 \mathrm{~nm}$ but was $550 \mathrm{~nm}$ in preadipocytes, whereas the emission maximum in adipocytes was $580 \mathrm{~nm}$ but was $630 \mathrm{~nm}$ in preadipocytes (Supplementary Fig. 2c and Fig. 2f). In adipocytes, the excitation and emission spectra of Nile red were blue-shifted, therefore increasing the overlap with the spectra of 2-NBDG. It is likely that this is due to the extensive lipid present in the adipocytes [13].

To further investigate this effect and determine if the observed spectral overlap precludes the selective detection of 2-NBDG and Nile red, we quantified the fluorescence of singly stained adipocytes in comparison to costained adipocytes with spectrofluorometry at precise wavelengths (Figure 3). At all wavelengths examined, the fluorescence intensity of the costained samples was greater than that of either of the singly stained samples. Likely owing to the relatively high intensity of Nile red compared to 2-NBDG, the profiles of the spectra from the costained samples closely resembled those from the Nile red-stained samples (Figures 3(b) $-3(\mathrm{f}))$.

However, when the fluorophores were excited at $480 \mathrm{~nm}$, there was a small region in the emission spectra between $500 \mathrm{~nm}$ and $525 \mathrm{~nm}$ where 2-NBDG emitted more intensely than Nile red (Figure 3(d) Inset). It is possible that detecting fluorescence in this region could lead to selective detection of 2-NBDG. We therefore quantified the fluorescence signals at these wavelengths using single point fluorescence measurements (Figures 4(a) and 4(b)). Emissions were not collected at $500 \mathrm{~nm}$ as the fluorophores' low intensity at this wavelength approached the instrument's lower limit of detection. At excitation and emission wavelengths $480 \mathrm{~nm}$ and $510 \mathrm{~nm}$, respectively, we found that the fluorescence from the costained preadipocytes was not significantly different from that of the 2-NBDG-stained preadipocytes (Figure 4(a)). We calculated the expected intensity of emission from the costained preadipocytes by adding the fluorescence detected from the single stained cells and found that the signal detected in the costained cells was not significantly different than what was expected (Figures 4(a) and 4(b)).

We found that the lipid-laden adipocytes stained significantly more intensely than the preadipocytes with both 2-NBDG and Nile red when emissions were collected at $510 \mathrm{~nm}$ and $520 \mathrm{~nm}$ (Figures 4(a) and 4(b)). As expected, 2NBDG fluorescence was significantly greater than Nile red fluorescence in singly stained cells; however, Nile red was still easily detectable in the adipocytes. The measured intensity of costained cells was significantly higher than the expected intensity suggesting a synergistic effect in the costained adipocytes when detected at $520 \mathrm{~nm}$.

To further explore whether Nile red can be selectively detected even when 2-NBDG is present, single point fluorescence measurements were made with singly stained and costained cells using wavelengths that we found to maximally excite Nile red (Figure 3). The costained adipocytes had significantly higher fluorescence intensities than both the Nile red singly stained samples and the computed sum of the 2-NBDG and Nile red fluorescence intensities (Figure 4(c)). Thus, in adipocytes, $2-\mathrm{NBDG}$ and Nile red costaining appears to synergistically increase the fluorescent intensity detected at wavelengths ideal for both $2-\mathrm{NBDG}$ and Nile red.

\section{Discussion}

Here, we show through both qualitative and quantitative fluorescence methods that 2-NBDG cannot be selectively detected in the presence of Nile red-stained lipid. Therefore, these fluorophores cannot be used simultaneously to measure glucose uptake and lipid accumulation in cells with abundant lipid.

We observed that the cellular structures detected by Nile red were different when using green or red emissions. As the diffuse red fluorescence in both the adipocytes and preadipocytes did not match the punctate morphology of the lipid droplets, we can conclude that Nile red is capable of detecting additional structures. These observations are in line with previous reports outlining the broad range of Nile red emissions $[15,23,24]$. It is suggested that solvatochromism is the source of this broad range as the fluorescence spectra shift abruptly in response to the polarity of the environment $[15,16]$. Therefore, our observation of diffuse Nile red staining is consistent with staining nonpolar structures outside of lipid droplets.

While the shift in Nile red fluorescence can be favourable, we observed that it caused substantial overlap with the 2NBDG spectra. In support of the microscopy and spectrofluorometry data shown here, we also performed a preliminary flow cytometry experiment with 2-NBDG and Nile red singly stained and costained adipocytes as flow cytometry can use compensation to reduce spillover from Nile red when detecting 2-NBDG. Similar to the results shown here, we found that the costained adipocytes were more fluorescent than the Nile red singly stained adipocytes and that this difference was greater than the fluorescence of the Nile red or 2-NBDG singly stained adipocytes (data not shown). Thus, even fluorescent compensation cannot reduce the spectral overlap enough to distinguish these fluorophores.

Similar to our findings when generating the spectra of the costained adipocytes, we were unable to find a wavelength that permitted selective detection of 2-NBDG even using precisely selected wavelengths. Previously, NBD-based fluorophores have been used simultaneously with Nile red, a method that we show to return false results. The authors used Nile red fluorescence to validate that NBD-sphingomyelin [18] or NBD-cholesterol $[12,17]$ colocalized to lipid droplets where they claim to have specifically excited and detected the NBD-based fluorophore in the presence of Nile red. There are only minor spectral differences between 2-NBDG and lipid-associated NBD-based fluorophores [25, 26]; therefore, these lipid-associated NBD-based fluorophores are detected with the same filter sets used to detect 2-NBDG. Thus, it is not clear that these authors were specifically detecting the NBD-based fluorophores in these experiments since the broad fluorescence of Nile red impairs the selective detection 


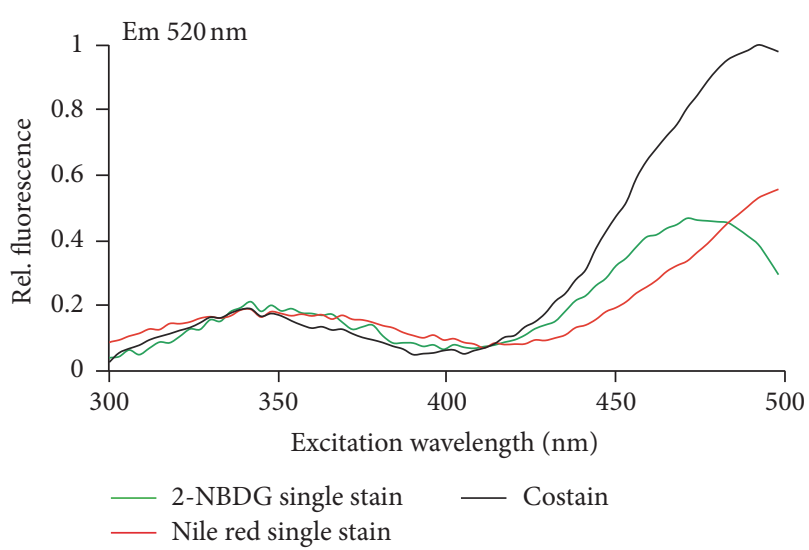

(a)

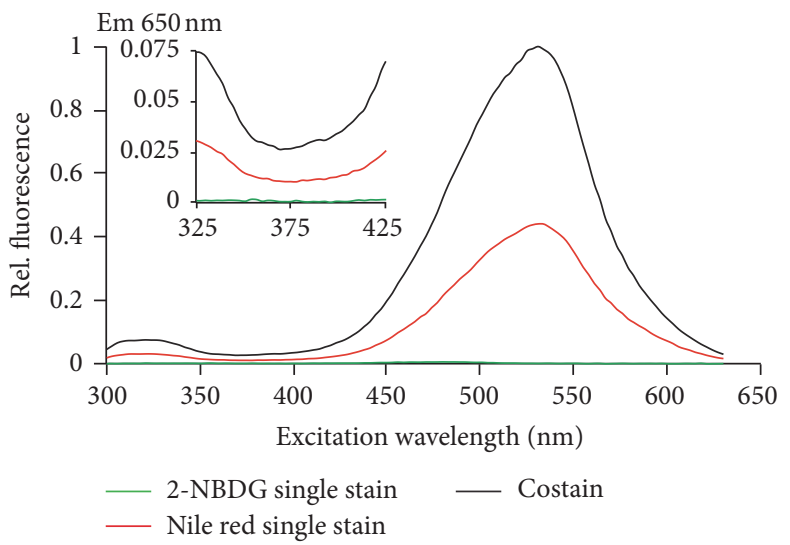

(c)

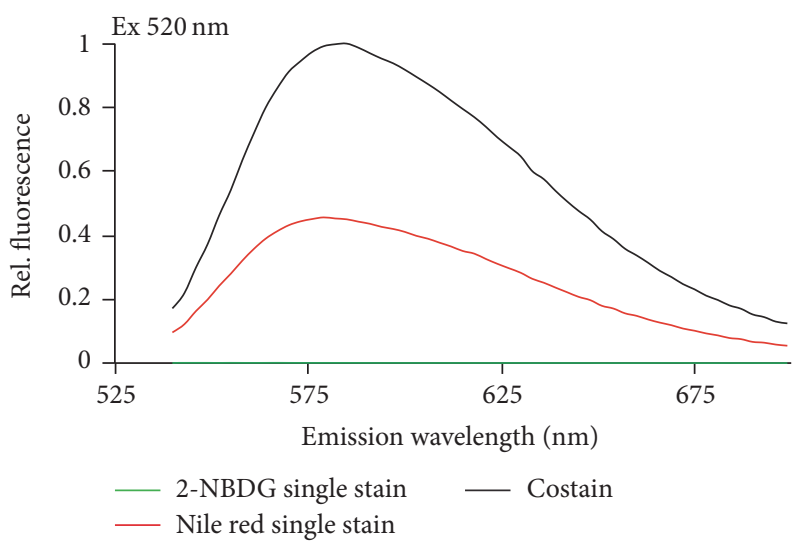

(e)

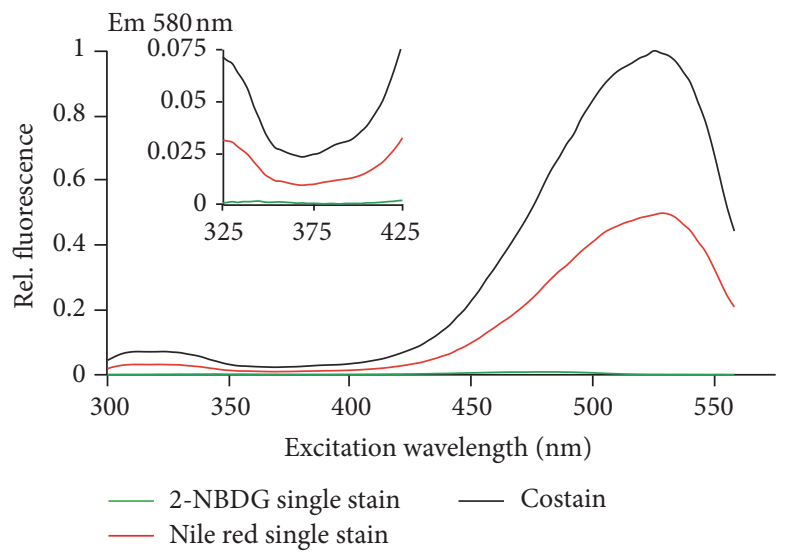

(b)

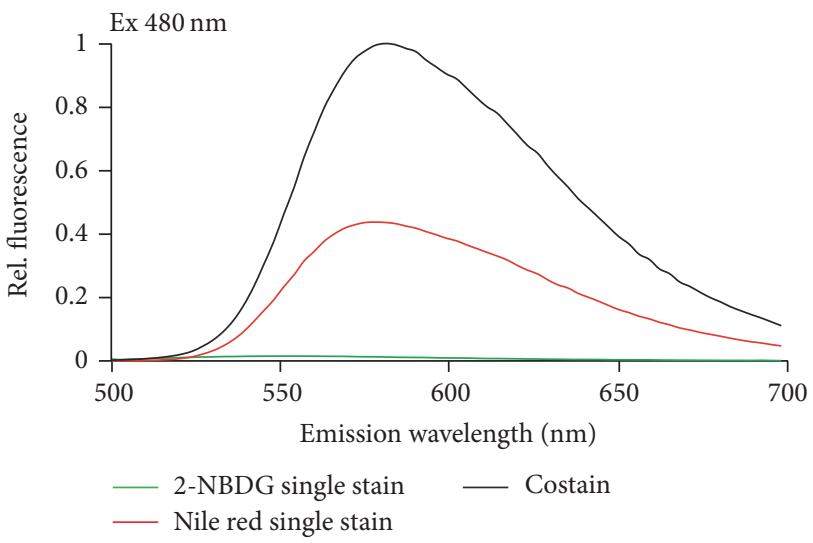

(d)

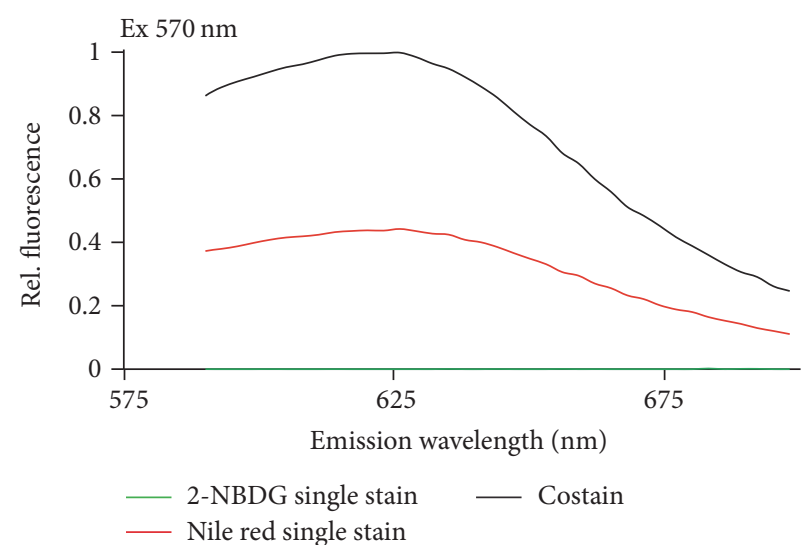

(f)

FIGURE 3: Comparison of the excitation and emission spectra of 2-NBDG and Nile red singly stained and costained 3T3-L1 adipocytes. Excitation spectra of samples with emissions detected at (a) $520 \mathrm{~nm}$, (b) $580 \mathrm{~nm}$, and (c) $650 \mathrm{~nm}$. Emission spectra of samples when excited at (d) $480 \mathrm{~nm}$, (e) $520 \mathrm{~nm}$, and (f) $570 \mathrm{~nm}$. Insets show the indicated region enlarged to highlight regions of potential selectivity when comparing single stained to costained adipocytes. The fluorescence intensity is shown as relative to the global maximum for each panel. Representative spectra from $n=3$ experiments.

of NBD-based fluorophores. The use of other green-emitting fluorophores such as GFP with Nile red [27] should also be carefully considered as the filters used for GFP detection are similar to those used for 2-NBDG and could thus detect Nile red.
It could be possible to first stain with and detect $2-\mathrm{NBDG}$ and then stain with and detect Nile red. This sequential staining method was used by Falchi et al. [19] where they first stained with 2-NBDG, fixed the cells to prevent organelle movement, visualized 2-NBDG, overstained with Nile red, 


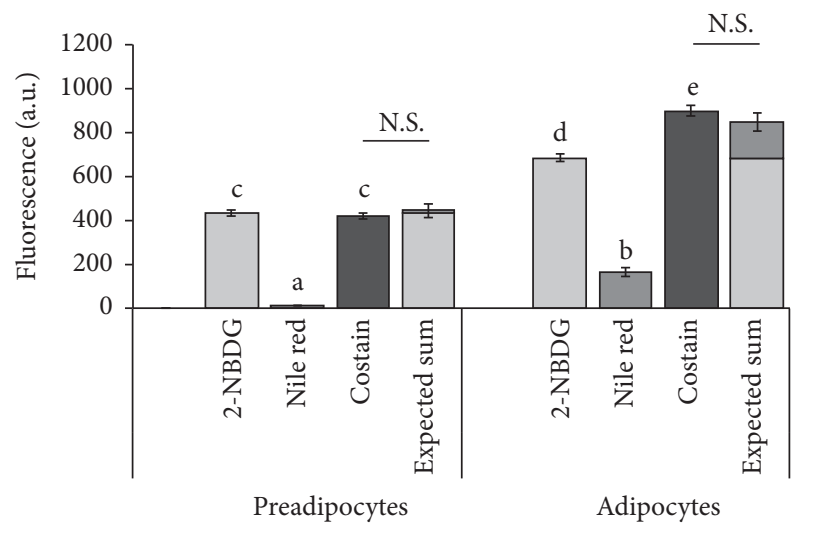

(a)

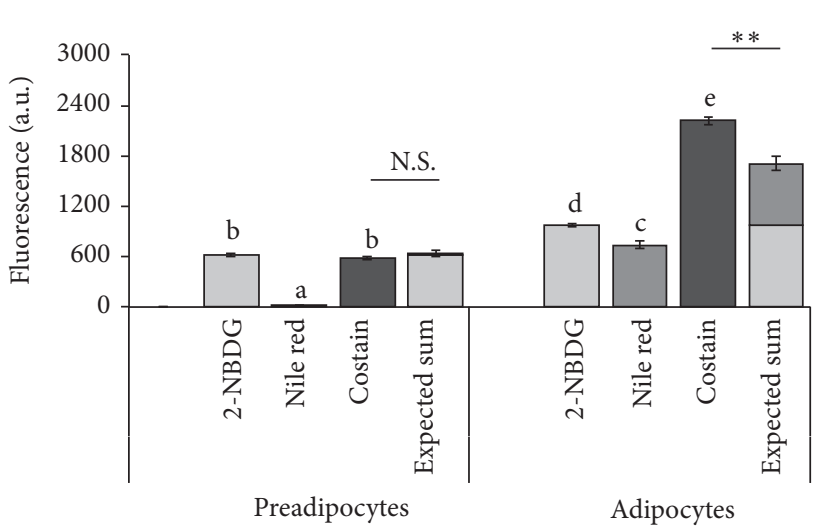

(b)

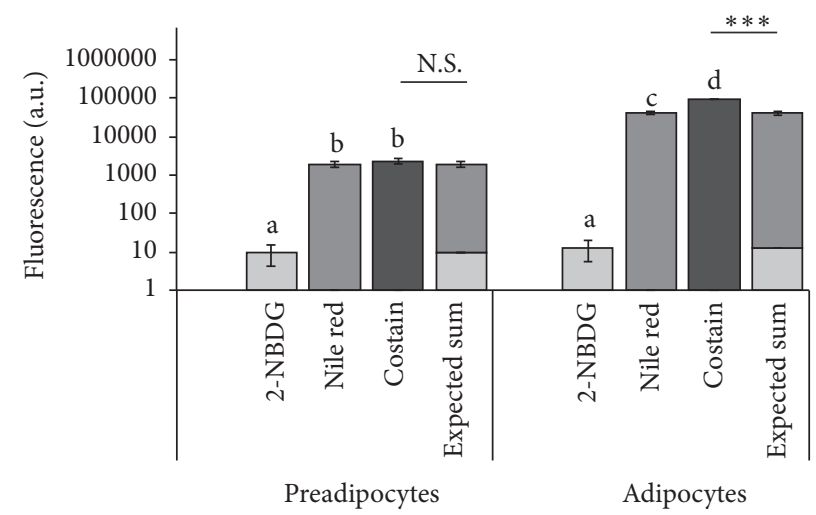

(c)

FIGURE 4: Quantification and statistical analysis of the fluorescence of cell stained with only 2-NBDG or Nile red compared to costained cells. (a) Fluorescent intensity with excitation and emission wavelengths set at $480 \mathrm{~nm}$ and $510 \mathrm{~nm}$, respectively, (b) $480 \mathrm{~nm}$ and $520 \mathrm{~nm}$, respectively, and (c) $520 \mathrm{~nm}$ and $580 \mathrm{~nm}$, respectively. Statistical significance was determined with a one-way ANOVA and Tukey's post hoc test. Different lower case letters indicate significant difference at $P<0.05$. The actual signal detected in the costained cells and the expected intensity (determined by summing the singly stained cells) were compared by Student's $t$-test. The values represent means \pm SD, $n=3$, ${ }^{* *} P<0.01 ;{ }^{* * *} P<0.001$.

and then visualized Nile red at longer wavelengths that do not detect 2-NBDG. To completely avoid the potential of fluorophore interactions, different fluorophores could be used with nonoverlapping spectra. There are currently no viable fluorescent conjugates of D-glucose other than 2NBDG; however, there are a variety of lipid droplet-selective stains. Of those that may potentially be used simultaneously with 2-NBDG, since they do not have substantially overlapping spectra, is the blue fluorophore 1,6-diphenyl-1,3,5hexatriene [28], the yellow fluorophore LD540 [29], and the red fluorophores HCS LipidTox ${ }^{\mathrm{TM}}$ Red and HCS LipidTox Deep Red [30].

\section{Conclusion}

Nile red and 2-NBDG are not compatible for the simultaneous detection of lipid and glucose due to substantial and synergistic spectral overlap of Nile red into the emission range of 2-NBDG, which is worsened by the solvatochromism of Nile red. Thus, we recommend the sequential use of 2-NBDG followed by Nile red for glucose and lipid detection or the use of other lipid dyes if simultaneous detection is required.

\section{Competing Interests}

The authors declare that they have no competing interests.

\section{Acknowledgments}

Funding was provided by Canadian Institute of Health Research (CIHR) and the Research and Development Corporation of Newfoundland and Labrador (RDC). Nikitha K. Pallegar is supported by a trainee award from the Beatrice Hunter Cancer Research Institute with funds provided by The Terry Fox Strategic Health Research Training Program in Cancer Research at CIHR and by Memorial University of Newfoundland. The authors gratefully acknowledge the resources provided by Dr. Hélène Paradis, Memorial University of Newfoundland, and the staff of the Medical Education 
and Laboratory Support Services at Memorial University of Newfoundland.

\section{References}

[1] W. N. P. Lee, L. O. Byerley, S. Bassilian et al., "Isotopomer study of lipogenesis in human hepatoma cells in culture: contribution of carbon and hydrogen atoms from glucose," Analytical Biochemistry, vol. 226, no. 1, pp. 100-112, 1995.

[2] Q. Wu, A. M. Ortegon, B. Tsang, H. Doege, K. R. Feingold, and A. Stahl, "FATP1 is an insulin-sensitive fatty acid transporter involved in diet-induced obesity," Molecular and Cellular Biology, vol. 26, no. 9, pp. 3455-3467, 2006.

[3] J. Law, I. Bloor, H. Budge, and M. E. Symonds, "The influence of sex steroids on adipose tissue growth and function," Hormone Molecular Biology and Clinical Investigation, vol. 19, no. 1, pp. 13-24, 2014.

[4] J. P. DeLany, Z. E. Floyd, S. Zvonic et al., "Proteomic analysis of primary cultures of human adipose-derived stem cells," Molecular and Cellular Proteomics, vol. 4, no. 6, pp. 731-740, 2005.

[5] A. T. Ali, W. E. Hochfeld, R. Myburgh, and M. S. Pepper, "Adipocyte and adipogenesis," European Journal of Cell Biology, vol. 92, no. 6-7, pp. 229-236, 2013.

[6] Q. Q. Tang and M. D. Lane, "Adipogenesis: from stem cell to adipocyte," Annual Review of Biochemistry, vol. 81, pp. 715-736, 2012.

[7] A. Soukas, N. D. Socci, B. D. Saatkamp, S. Novelli, and J. M. Friedman, "Distinct transcriptional profiles of adipogenesis in vivo and in vitro," The Journal of Biological Chemistry, vol. 276, no. 36, pp. 34167-34174, 2001.

[8] K. Yoshioka, H. Takahashi, T. Homma et al., "A novel fluorescent derivative of glucose applicable to the assessment of glucose uptake activity of Escherichia coli," Biochimica et Biophysica Acta, vol. 1289, no. 1, pp. 5-9, 1996.

[9] M. Smilkstein, N. Sriwilaijaroen, J. X. Kelly, P. Wilairat, and M. Riscoe, "Simple and inexpensive fluorescence-based technique for high-throughput antimalarial drug screening," Antimicrobial Agents and Chemotherapy, vol. 48, no. 5, pp. 1803-1806, 2004.

[10] M. Hall, I. Kazakova, and Y.-M. Yao, "High sensitivity immunoassays using particulate fluorescent labels," Analytical Biochemistry, vol. 272, no. 2, pp. 165-170, 1999.

[11] K. Yamada, M. Nakata, N. Horimoto, M. Saito, H. Matsuoka, and N. Inagaki, "Measurement of glucose uptake and intracellular calcium concentration in single, living pancreatic $\beta$-cells," Journal of Biological Chemistry, vol. 275, no. 29, pp. 2227822283, 2000.

[12] A. Frolov, A. Petrescu, B. P. Atshaves et al., "High density lipoprotein-mediated cholesterol uptake and targeting to lipid droplets in intact L-cell fibroblasts. A single- and multiphoton fluorescence approach," Journal of Biological Chemistry, vol. 275, no. 17, pp. 12769-12780, 2000.

[13] G. Diaz, M. Melis, B. Batetta, F. Angius, and A. M. Falchi, "Hydrophobic characterization of intracellular lipids in situ by Nile Red red/yellow emission ratio," Micron, vol. 39, no. 7, pp. 819-824, 2008.

[14] D. L. Sackett and J. Wolff, "Nile red as a polarity-sensitive fluorescent probe of hydrophobic protein surfaces," Analytical Biochemistry, vol. 167, no. 2, pp. 228-234, 1987.

[15] P. Greenspan, E. P. Mayer, and S. D. Fowler, "Nile red: a selective fluorescent stain for intracellular lipid droplets," Journal of Cell Biology, vol. 100, no. 3, pp. 965-973, 1985.
[16] P. Greenspan and S. D. Fowler, "Spectrofluorometric studies of the lipid probe, nile red," Journal of Lipid Research, vol. 26, no. 7, pp. 781-789, 1985.

[17] B. P. Atshaves, O. Starodub, A. McIntosh et al., "Sterol carrier protein-2 alters high density lipoprotein-mediated cholesterol efflux," Journal of Biological Chemistry, vol. 275, no. 47, pp. 36852-36861, 2000.

[18] A. L. McIntosh, S. M. Storey, and B. P. Atshaves, "Intracellular lipid droplets contain dynamic pools of sphingomyelin: ADRP binds phospholipids with high affinity," Lipids, vol. 45, no. 6, pp. 465-477, 2010.

[19] A. M. Falchi, B. Battetta, F. Sanna et al., "Intracellular cholesterol changes induced by translocator protein $(18 \mathrm{kDa}) \mathrm{TSPO} / \mathrm{PBR}$ ligands," Neuropharmacology, vol. 53, no. 2, pp. 318-329, 2007.

[20] N. C. Smith, N. A. Fairbridge, N. K. Pallegar, and S. L. Christian, "Dynamic upregulation of CD24 in pre-adipocytes promotes adipogenesis," Adipocyte, vol. 4, no. 2, pp. 89-100, 2015.

[21] C. A. Schneider, W. S. Rasband, and K. W. Eliceiri, "NIH Image to ImageJ: 25 years of image analysis," Nature Methods, vol. 9, no. 7, pp. 671-675, 2012.

[22] The R Core Team, $R$ : A Language an Environment for Statistical Computing, 2011.

[23] B. Xie, D. Stessman, J. H. Hart et al., "High-throughput fluorescence-activated cell sorting for lipid hyperaccumulating Chlamydomonas reinhardtii mutants," Plant Biotechnology Journal, vol. 12, no. 7, pp. 872-882, 2014.

[24] L. L. Listenberger and D. A. Brown, "Fluorescent detection of lipid droplets and associated proteins," Current Protocols in Cell Biology, no. chapter 24: unit 24.2., 2007.

[25] S. Fery-Forgues, J.-P. Fayet, and A. Lopez, "Drastic changes in the fluorescence properties of NBD probes with the polarity of the medium: involvement of a TICT state?" Journal of Photochemistry and Photobiology A: Chemistry, vol. 70, no. 3, pp. 229243, 1993.

[26] M. Amaro, H. A. Filipe, J. P. Prates Ramalho, M. Hof, and L. M. Loura, "Fluorescence of nitrobenzoxadiazole (NBD)-labeled lipids in model membranes is connected not to lipid mobility but to probe location," Physical Chemistry Chemical Physics, vol. 18, no. 10, pp. 7042-7054, 2016.

[27] S. Mishra, R. Khaddaj, S. Cottier, V. Stradalova, C. Jacob, and R. Schneiter, "Mature lipid droplets are accessible to ER luminal proteins," Journal of Cell Science, vol. 129, no. 20, pp. 3803-3815, 2016.

[28] S. Kaushik and A. M. Cuervo, "Degradation of lipid dropletassociated proteins by chaperone-mediated autophagy facilitates lipolysis," Nature Cell Biology, vol. 17, no. 6, pp. 759-770, 2015.

[29] J. Spandl, D. J. White, J. Peychl, and C. Thiele, "Live cell multicolor imaging of lipid droplets with a new dye, LD540," Traffic, vol. 10, no. 11, pp. 1579-1584, 2009.

[30] J. G. Granneman, H.-P. H. Moore, E. P. Mottillo, and Z. Zhu, "Functional interactions between Mldp (LSDP5) and Abhd5 in the control of intracellular lipid accumulation," The Journal of Biological Chemistry, vol. 284, no. 5, pp. 3049-3057, 2009. 

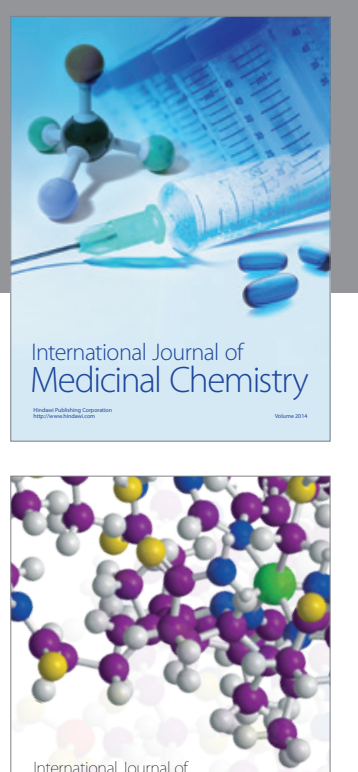

Carbohydrate Chemistry

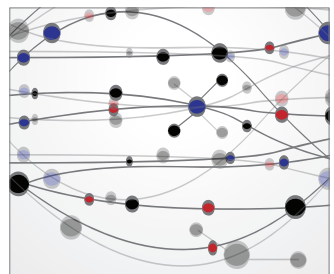

The Scientific World Journal
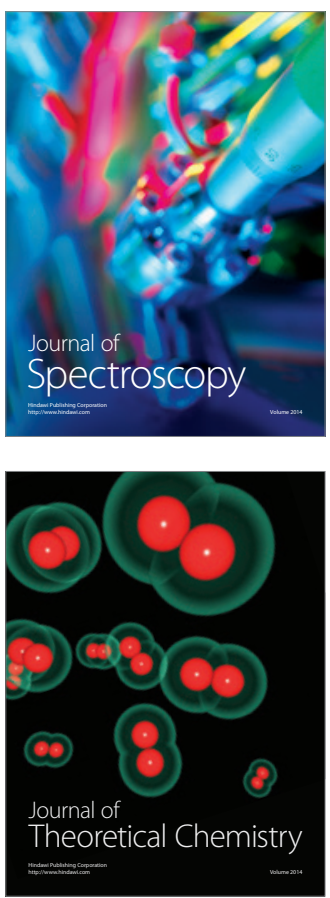
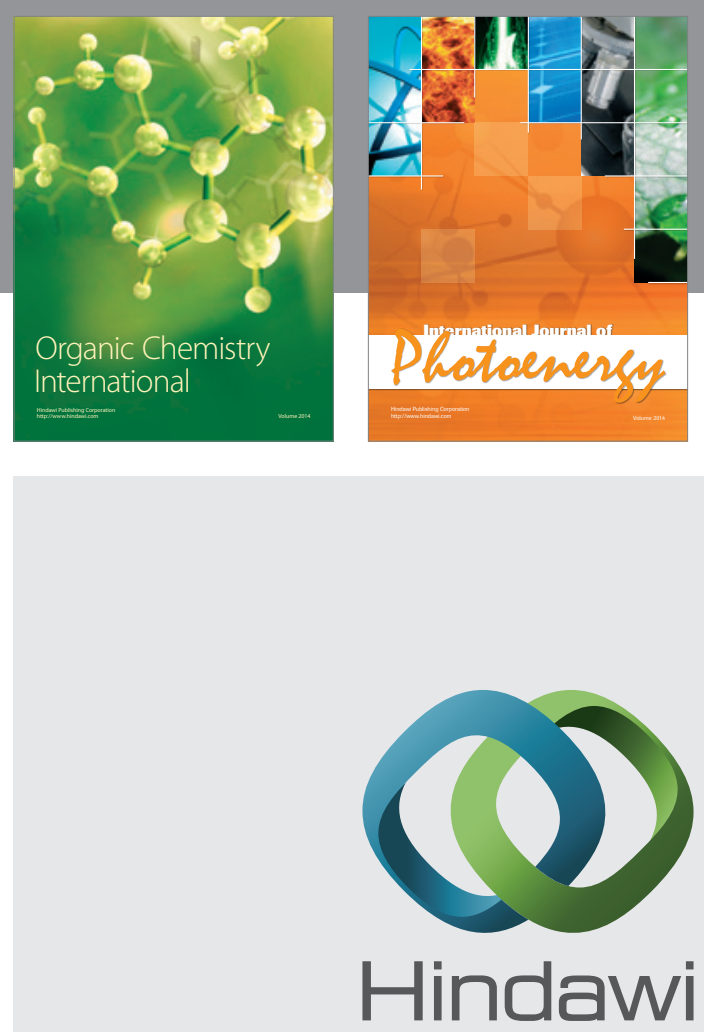

Submit your manuscripts at

http://www.hindawi.com

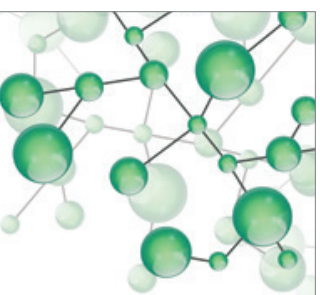

International Journal of

Inorganic Chemistry

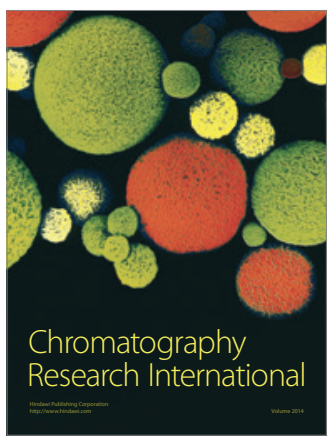

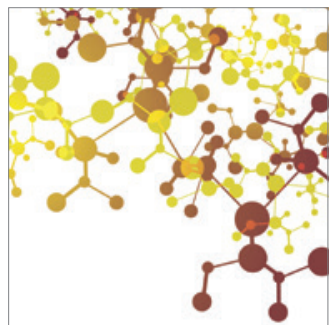

Applied Chemistry
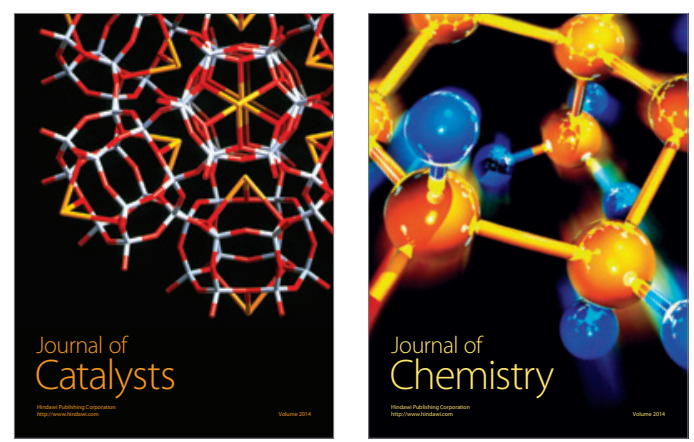
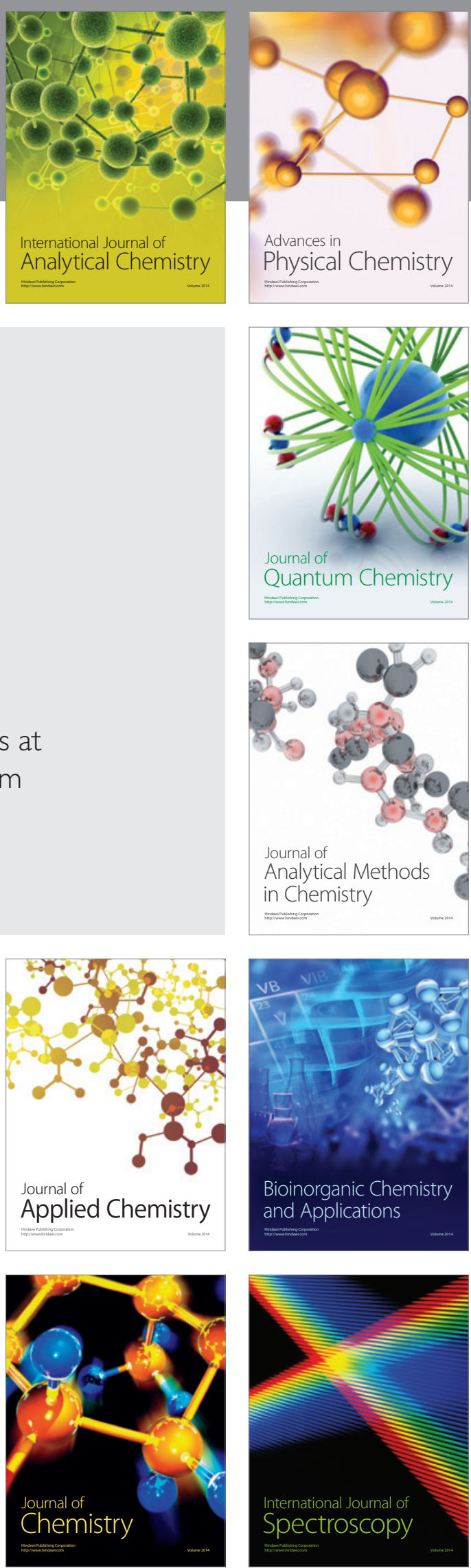